\title{
Mucoadhesive Microsphere Drug Delivery System (MMDDS): A Mini Review
}

\author{
Srilatha Malvey,*, A. Kottai Muthu², K. Balamurugan², J. Venkteshwar Rao ${ }^{1}$, Goverdhan Puchcha- \\ kayala $^{3}$, E. Ravinder ${ }^{4}$, Naresh Kshirasagar ${ }^{5}$ \\ ${ }^{1}$ Talla Padmavathi College of Pharmacy, Kareemabad Warangal, Telangana, India. \\ ${ }^{2}$ Annamalai University, FEAT, Annamalainagar, Tamilnadu, India. \\ ${ }^{3}$ Department of Pharmacological and Pharmaceutical Science, University of Houston, Houston, Texas, USA. \\ ${ }^{4}$ Vaagdevi College of Pharmacy, Hanamkonda, Telangana State, India. \\ ${ }^{5}$ Sree Dattha Institute of Pharmaceutical Science, Ibrahimpatnam, RR District, Telangana, India.
}

How to cite this paper: Srilatha Malvey, A. Kottai Muthu, K. Balamurugan, J. Venkteshwar Rao, Goverdhan Puchchakayala, E. Ravinder, Naresh Kshirasagar. (2021) Mucoadhesive Microsphere Drug Delivery System (MMDDS): A Mini Review. International Journal of Clinical and Experimental Medicine Research, 5(4), 435-444. DOI: 10.26855/ijcemr.2021.10.003

Received: July 6, 2021

Accepted: August 2, 2021

Published: September 9, 2021

*Corresponding author: Srilatha Malvey, Talla Padmavathi College of Pharmacy, Kareemabad Warangal, Telangana, India.

Email: nareshvcop@gmail.com

\begin{abstract}
Over the past few decades, broad research work has been carried out to develop a gastro-retentive dosage form, this type of dosage form shows effectiveness in delivery of drug due to its presence in the stomach for enough time. The present survey is concern about the review of bioadhesive or mucoadhesive microspheres, for its combined effect of bioadhesion and surface to volume ratio of microspheres in which particle size is range from 1-1,000 $\mu \mathrm{m}$ range in diameter having a core of drug and outer layer of mucoadhesive polymer. Mucoadhesive microspheres have advantage of controlled and sustained release of drug from dosage form with less fluctuation in blood concentration. Evaluation of Mucoadhesive Microspheres has to be carried for FTIR Studies, Scanning Electron Microscopy (SEM), Micrometric Studies, Bulk Density, Tapped Density, Angle of Repose, \% Compressibility Index, Hausner's Ratio, Particle Size, Density Determination, Floating Behavior, Angle of Contact, Swelling Index, Percentage Yield, Percentage Drug Content, The Percentage of Drug Entrapment, Drug Entrapment Efficiency, Ex Vitro Mucoadhesive Study, In Vitro Drug Release Studies, In Vivo X-Ray Imaging Study, In Vitro Wash-Off Test for Microspheres, Mucoadhesive Microspheres Stability in the $\mathrm{pH} 1.0 \mathrm{HCl}$ Medium, Stability Studies. The present review highlights some of the new drugs that can be benefits from MMDDS.
\end{abstract}

\section{Keywords}

Mucoadhesive, Microspheres, Sustained Release

\section{Introduction}

The most convenient route of drug administration is an oral route with a high rank of patient compliance, due to its ease of administration, sustained or controlled release drug delivery system provides drug release, and this dosage form is well suitable for the drugs having short half-life. For the development of oral sustained or controlled drug delivery system, one of the main tasks is to modify the Gastro Intestinal (GI) transits time, normally gastric resistant time is 2-3 hr. It may depend upon the individual to individual called as intersubjective variability and fed, fasted state of the stomach [1].

Microspheres constitute a significant part of these particulate drug delivery systems for its small size and having efficient carrier capacity. Microspheres are the carrier linked drug delivery system in which inner core of active 
moiety and outer layers of a polymer as a coating material. However, the success of these microspheres is limited due to their short residence time at the site of absorption. It would be advantageous to have resources for providing an intimate contact of the drug delivery system with the absorbing membrane, this can be achieved by developing "Mucoadhesive Microspheres". Mucoadhesive microspheres have advantages like efficient absorption and enhanced bioavailability of various drugs due to a high surface to volume ratio, due to close contact with the mucus layer and precise targeting of drugs to the absorption site. One requisite for the effective performance of oral controlled or sustained drug delivery is that the drug should have good absorption throughout the gastrointestinal tract, preferably by passion diffusion mechanism.

The rational for development of oral gastro-retentive mucoadhesive microspheres dosage form is fundamental for a drug that is adsorbed from the stomach which is tremendously degraded in stomach enzymes, it can alter high dose fluctuation in blood, reduce the risk of resistant to bacteria especially in the case of antibiotic. Drugs that have poor bioavailability due their absorption is restricted to the upper GI tract can be delivered efficiently thereby maximizing their absorption and improving their absolute bioavailability [2].

\subsection{Factors are considered in drug delivery}

The gastric $\mathrm{pH}$ is an important factor taken into consideration, which shows influence on the performance of an action of drug absorption. The gastric $\mathrm{pH}$ is not constant throughout the GIT, which are influence by various factors such as diet, high protein or carbohydrates intake diseases, the presence of gases and other fermented products. Gastric emptying is another important parameter to consider, it depends upon meal taken in quantity. Gastric emptying occurs during fasting as well as in fed states, in fasted states the electrical motion of the stomach was controlled by some cyclic contractile proceedings commonly known as migrating myoelectric complexes (MMC). There are four phases of activity in MMC Phase I, II, III, IV, these cycles are initiated by feeding which regulates contractile activity which may appears for 3-4 hr. Thus, feeding in frequency manner may prolong gastric retention time. A subject/ individual under stress shows increased in gastric emptying rate while, depression slows it. In general, women without physical activity has slower emptying rate than young with high physical activity respectively in addition sleeping body posture may influence the gastric emptying [3].

Most of the drug adsorption takes place in stomach and upper part of the intestine, as conventional tablet do not possess the resistance or mucoadhesive behavior of dosage form. Therefore, they cannot be released in their colon. In order to overcome this problem in conventional dosage forms are designed as a novel drug delivery system called mucoadhesive drug delivery system approach.

Additional advantage added in their novel dosage form [5].

$>$ An upgrading in therapeutics effectiveness.

A decline in drug loss.

Reduction in toxicity.

$>$ Decline in high drug fluctuation in plasma concentration and maintained by sustained drug release.

$>$ Superior to single-unit floating dosage form, such as mucoadhesive microsphere releases drug uniformly and there is no risk of dose dumping.

$>$ Benefits to the drug that act locally in the stomach called as site-specific drug delivery.

$>$ Better therapeutics effect for short half-life drugs.

\subsection{Physiology of stomach}

Stomach is used as depot for sustained or controlled release dosage forms, the stomach is dived into three parts

1) Fundus

2) Body

3) Pylorus

The proximal stomach made up of the fundus and body region serve as a reservoir for ingested material, while the digital region that is pylorus is the mixing sieve the process of the gastric emptying occurs both during fasting and fed stated differ in MMC. Scintigraphically studies help in measurement of gastric emptying rate in healthy human subject. The mucosal surface area in the body far 100X greater than the skin in the intestinal area. There are many advantages to the development of mucoadhesive drug delivery system.

\section{Basics of Gastric Retention}

Various approaches have been pursued to increases the retention of an oral dosage form in the stomach mucoadhesive system, swelling expanding, bioadhesion, modulated dosage form other specific devices [1], FDDS or hy- 
drodynamically balanced system has a bulk density lower than gastric fluid and thus remain buoyant in the stomach without affecting the gastric emptying rate for a prolonged period. While dosage form is mucoadhesive on the stomach the drug is released slowly in a sustained manner, which leads to release of drug into systemic circulation without major fluctuation in plasma in the case of swelling and expanding dosage form it prevents entering into intestine, which remains in stomach for prolonged period of time shows sustained action of the drug.

\subsection{Characteristics of an ideal mucoadhesive polymer}

It should be nonirritant to the mucous membrane.

It should preferably form a strong no covalent bond with the mucin-epithelial cell surfaces.

It should adhere rapidly to tissue and should have some site specificity.

It should allow easily integrated into the drug and should offer no hindrance to its release.

The polymers must not decompose on storage of the dosage form and its shelf life.

The cost of the polymer should not be high so that the prepared dosage form remains competitive.

It should be nonabsorbable from GI tract.

Robinson and his group using the fluorescence technique concluded that:

Cationic and anionic polymers bind more effectively than neutral polymers.

Polyanions are better than polycations in terms of binding/potential toxicity, and further, that water-insoluble polymers give greater flexibility in dosage form design compared with rapidly or slowly dissolving water-soluble polymers.

$>\quad$ Anionic polymers with sulfate groups bind more effectively than those with carboxylic groups. iv. A degree of binding is proportional to the charge density on the polymer.

Highly binding polymers include carboxymethylcellulose, gelatin, hyaluronic acid, carbopol, and polycarbophil.

\section{Mechanism of Mucoadhesion}

The mucosal layer lines the gastrointestinal tract, acts as a lubricating and protective barrier for eye, mouth, nasal cavity, vaginal cavity, mucus is constantly secreted by goblet cells [2]. Mucoadhesive properties of mucus are due to the existence of glycoprotein called mucins are extremely glycosylated large molecules, which forms negativecharge at physiological pH 7.4. In general, there are theories explain the adhesion to the mucosal surface [3], electronic theory, wetting theory, adsorption theory, diffusion theory, fracture theory, mechanical theory, the combination all the theories result in adhesion (Smart, 2005). There are two stages of adhesion, wetting stage is the intimate contact between the polymers, \& mucosal surface, which is required for mucoadhesion, that is a polymer, will be exposed to the mucus and wetting occurs, the polymer forms a non-covalent bond with mucus, which is due to the presence of hydrophilic group on the polymer. The extent of the swelling of the polymer depends upon the contact time of polymer with mucus and penetration of the polymer chain into the mucosal surface is often directed by the initial contact time.

Consolidation stage was a mucoadhesive strength is stronger when the polymer itself is completely dehydrated and are placed on the mucosal surface. The polymer will begin to absorb the moisture from the mucosal layer, which once dehydrated switches from having lubricating properties to adhesive properties.

\section{Factors Affecting Mucoadhesion}

\subsection{Charge on the polymer}

Commonly used polymers have either an anionic or cationic charged some non-ionic polymers are also used, strong anion on the polymer is good sign of mucoadhesion, it has been shown that some cationic polymers are likely to found higher mucoadhesive property particularly in a neutral or slightly alkaline medium [4].

\section{$4.2 \mathrm{pH}$}

$\mathrm{pH}$ of the medium is important parameter for the degree of hydration. Bioadhesion can be influenced by the charges present on the surface of mucus as well as certain ionisable bioadhesive polymers. Mucus will have a different charge density depending on $\mathrm{pH}$ due to difference in dissociation of functional groups on the carbohydrate moiety and the amino acids of the polypeptide backbone [4]. 


\subsection{Molecular weight}

The optimum molecular weight for maximum mucoadhesion depends upon the type of mucoadhesive polymer and tissue, low molecular polymer penetrate the mucus layer better than high molecular weight, for example, polyethylene glycol with a molecular weight of 200Daltons has little adhesive character whereas PEG with 4000Daltons molecular weight has better and PEG with 6000Dalton has superior adhesive properties [4].

\subsection{Swelling}

Once the polymer has adhered to the mucosal layer, it starts absorbing moisture from the surface and swell. This is advantageous as the surface area between the polymer and the mucosal layer will increase, by modifying the polymer with the crosslinking moieties, such as the introduction of thiol groups the degree of swelling can be controlled [4].

\subsection{Mucoadhesive polymers}

Majority of polymers used in mucoadhesive are hydrophilic in nature, which shows better adhesion properties but shorter duration of action when compared to the synthetic polymer [5].

Mucoadhesive polymers are water-soluble and water-insoluble polymers, which are swellable networks, joined by cross-linking agents. These polymers have optimal polarity to permit sufficient wetting by the mucus and optimal fluidity that permits the mutual adsorption and interpenetration of polymer and mucus to take place. Mucoadhesive polymers that adhere to the mucin-epithelial surface conveniently divided into three broad classes.

1) Polymers that becomes sticky, when placed in water and owe their mucoadhesion to stickiness.

2) Polymers that adhere to non-specific, non-covalent interactions that is primarily electrostatic in nature (although hydrogen and hydrophobic bonding may be significant).

3) Polymers that bind to the specific receptor site. All three polymers types can use for drug delivery.

\subsection{Chitosan}

Derived from the deacetylation of chitin is the most commonly used material for the mucoadhesive polymer. It is naturally occurring polysaccharides, which is the cationic, and hydrophilic in nature, cationic polymer is a unique character of chitosan as it attaches to the negatively charged mucosal membrane. Chitosan is a biocompatible and biodegradable amino polysaccharide, which is soluble in aqueous solutions at $\mathrm{pH}$ less than 6.5 [6].

It has been widely used for developing drug delivery system because of its excellent mucoadhesive, the nature of the interaction between chitosan and mucin remains poorly defined.

\subsection{Polyacrylic acid (PAA)}

PAA is an anionic polymer. The core method of adhesion of anionic polymer is due to hydrogen bonding between the polymer and mucin. Contacts of PAA with mucin are strongest at an acidic $\mathrm{pH}$, indicating that the polymer has mucoadhesive property in its protonated state.

\subsection{Pectin}

Similar to chitosan, pectin is a polysaccharide isolated from the citrus peel or apple. This natural of polymer has non-toxic properties and often used within the food and pharmaceutical industries. The Pectin is an anionic, complex heteropolymer, which contains residuals of galacturonate and rhamnose and has the high level of carboxyl group along with its backbone. The swelling nature of a polymer is due to the presence of hydroxy groups. Modification of pectin backbone methoxylated or amidated carboxyl group and the degree of modification can allow for the altered level of mucoadhesion. Incorporation of solid, liquid or gases into polymeric coatings done by microencapsulation technique. The different methods used for various microspheres preparation depends on particle size, route of administration, duration of drug release and these above characters related to rpm, the method of cross-linking, aconcentration of polymer, evaporation time, co-precipitation etc.

\section{Methods of Preparations}

\subsection{Phase separation coacervation technique}

This development will take on the principle of decreasing the solubility of the polymer in the organic phase to mark the formation of polymer rich phase called the coacervates. In this method, the drug particles could distribute 
in a solution of the polymer system, which makes the first polymer to phase separate and engulf the drug particles. Addition of non-solvent effects in the solidification of the polymer. Polylactic acid (PLA) microspheres prepared by this method by using butadiene as incompatible polymer. The process variables are very important in the rate of achieving the coacervates determines the distribution of the polymer film, the particle size, and agglomeration of the formed particles. The agglomeration must be circumvented by stirring the suspension using a suitable speed stirrer since as the process of microspheres formation begins the formed polymerize globules start to stick and form the agglomerates. Therefore, the process variables are critical as they control the kinetics of the formed particles since there is no defined state of equilibrium attainment [7].

\subsection{Emulsion cross-linking method}

In this method, drug is dissolved in aqueous gelatin solution, which was formerly heated for $1 \mathrm{hr}$ at $40^{\circ} \mathrm{C}$. The solution is added dropwise to liquid paraffin while stirring the mixture at $1,500 \mathrm{rpm}$ for $10 \mathrm{~min}$ at $35^{\circ} \mathrm{C}$, results in w/o emulsion then further stirring is done for $10 \mathrm{~min}$ at $15^{\circ} \mathrm{C}$ Thus the formed microspheres are washed for three times with acetone and isopropyl alcohol, which then air dried and dispersed in $5 \mathrm{~mL}$ of aqueous glutaraldehyde saturated toluene solution at room temperature for 3 hrs. for cross-linking and then treated with $100 \mathrm{~mL}$ of $10 \mathrm{~mm}$ glycine solution containing $0.1 \% \mathrm{w} / \mathrm{v}$ of tween 80 at $37^{\circ} \mathrm{C}$ for $10 \mathrm{~min}$ to block unreacted glutaraldehyde.

\subsection{Solvent Evaporation}

The processes are conceded out in a liquid manufacturing vehicle. The microcapsule coating is dispersed in a volatile solvent which is immiscible with the liquid manufacturing vehicle phase. A core material to be microencapsulated is dissolved or dispersed in the coating polymer solution with agitation the core material mixture is dispersed in the liquid manufacturing vehicle phase to obtain the appropriate size microcapsule. The mixture is then heated if necessary to evaporate the solvent for the polymer of the core material is dispersed in the polymer solution, polymer shrinks around the core. If the core material is dissolved in the coating polymer solution, matrix-type microcapsules are formed. The core materials may be either water-soluble or water in-soluble materials. Solvent evaporation involves the formation of an emulsion between polymer solution and an immiscible continuous phase whether aqueous $(\mathrm{o} / \mathrm{w})$ or non-aqueous. The comparison of mucoadhesive microspheres of hyaluronic acid, Chitosan glutamate and a combination of the two prepared by solvent evaporation with microcapsules of hyaluronic acid and gelatin prepared.

\subsection{Ionic gelation}

Alginate/chitosan particulate system for diclofenac sodium release was prepared using this technique. In this method, drug is added to aqueous solution of sodium alginate. In order to get the complete solution stirring is continued and after that it is added dropwise to a solution containing $\mathrm{Ca} 2+/ \mathrm{Al} 3+$. Microspheres that are formed were kept in original solution for $24 \mathrm{hr}$. for internal gelification followed by filtration for separation. The complete release is obtained at $\mathrm{pH}$ 6.4-7.2 but the drug will not release in acidic $\mathrm{pH}$ [7].

\subsection{Spray Drying}

In spray drying, the polymer is first dissolved in a suitable volatile organic solvent such as dichloromethane, Acetone, etc. The drug in the solid form is then dispersed in the polymer solution under high-speed homogenization. This dispersion is then atomized in a stream of hot air. The atomization leads to the formation of the small droplets or the fine mist from which the solvent evaporates instantaneously leading the formation of the microspheres in a size range $1-100 \mu \mathrm{m}$. Microparticles should be separated from the hot air by means of the cyclone separator while the trace of solvent was removed by vacuum drying. One of the major advantages of the process is the feasibility of operation under aseptic conditions. This process is quick and this leads to the formation of porous microparticles [8].

\subsection{Multiple emulsion polymerization technique}

Multiple emulsion method involves the formation of (o/w) Primary emulsion (nonaqueous drug solution in a polymer solution) and the addition of primary emulsion to external oily phase to form o/w/o emulsion followed by either addition of cross-linking agent (glutaraldehyde) and evaporation of the organic solvent. This method of preparation is ideal for incorporating poorly aqueous soluble drug, thus enhancing its bioavailability [9]. 


\subsection{Orifice-Ionic Gelation Method}

Sodium alginate and the mucoadhesive polymer are dispersed in purified water $(50 \mathrm{ml})$ to form a homogeneous polymer mixture. A drug is added to the polymer matrix and mixed thoroughly to form a smooth viscous dispersion. Resulting dispersion is then sprayed into calcium chloride $(10 \% \mathrm{w} / \mathrm{v})$ solution by continuous stirring. Produced droplets are retained in the calcium chloride solution for 15 minutes to complete the curing reaction and to produce rigid spherical microspheres. The resulting microspheres is collected by decantation, and the product thus separated is washed repeatedly with purified water to remove excess calcium impurity deposited on the surface of microspheres and then dried at $45^{\circ} \mathrm{C}$ for $12 \mathrm{hrs}$ [10].

\section{Evaluation of Mucoadhesive Microspheres}

\subsection{FTIR studies}

FTIR studies should be done for possible interaction between the drug and polymers in the position of absorption bands [11].

\subsection{Scanning electron microscopy (SEM)}

The surface topography of the microspheres should be examined using scanning electron microscopy at different magnification, the acceleration voltage applied samples were mounted on a scanning electron microscopy holder using a double-sided adhesive tape and coated with gold-palladium under vacuum, and the surface topography was investigated. The external and internal morphology of the microspheres is studied by (SEM) [12].

\subsection{Micrometric studies}

Mucoadhesive microsphere is evaluated as per the method described by Aulton the angle of repose and flow rate is determined by the funnel method. The bulk density and tapped density were obtained by the cylinder method. [13].

\subsection{Bulk density}

The bulk density of a powder is the ratio of the mass of an untapped powder sample and its volume containing the contribution of the interparticulate void spaces. Hence, the bulk density depends on both the density of powder particles and the spatial arrangement of particles in the powder bed. The bulking properties of a powder are dependent upon the preparation, treatment, and storage of the sample, the bulk density is expressed in grams per milliliter $(\mathrm{g} / \mathrm{ml})$

$$
\text { Bulk density }\left(\rho_{\mathrm{b}}\right)=\mathrm{W} / \mathrm{V}_{\mathrm{b}}
$$

Where $\mathrm{w}$ is weight of the sample in grams, $\mathrm{V}_{\mathrm{b}}$ is final bulk volume of granules in $\mathrm{cm}^{3}$

\subsection{Tapped density}

The tapped density is an increased bulk density attained after mechanically tapping a container containing the powder sample. The tapped density is obtained by mechanically tapping a graduated measuring cylinder or vessel containing the powder sample. After observing the initial powder volume or mass, the measuring cylinder, and volume or mass readings are taken, if the volume changes were observed more than 2 percentages. The mechanical tapping is further continued to obtain the constant reading. Finally, tapped density can be calculated by the following equation [14].

$$
\text { Tapped density }\left(\rho_{t}\right)=\mathrm{W} / \mathrm{V}_{\mathrm{t}}
$$

\section{Angle of repose}

The fixed funnel method was used for estimating the angle of repose for microspheres.

$$
\Theta=\tan ^{-1}(\mathrm{~h} / \mathrm{r})
$$

Where $\Theta$ is the angle of repose, his the height of the cone, and $r$ is the radius of cone or base radius [15].

\section{\% Compressibility index}

Percentage compressibility Index $=1-\{\mathrm{V} / \mathrm{Vo}\} \times 100$ where $\mathrm{V}$ and $\mathrm{V}_{\mathrm{o}}$ are the volumes of the sample after and before the standard tapping, respectively [16]. 


\section{Hausner's ratio}

The Hausner ratio is a number that is correlated to the flowability of a powder or granular material It can be calculated \& expressed by the following equation [16].

$$
\mathrm{H}=\rho_{\mathrm{b} / \rho_{\mathrm{t}}}
$$

\section{Particle size}

Size is measured using an optical microscope, and mean particle is calculated by can be measured by the optical microscopy method using a calibration stage micrometer. Further particle size analysis can be done by fitting camera imaging accessory to the microscope [16]. Different sizes of microspheres and their distribution in each batch are measured by sieving in a mechanical shaker, using a standard sieve (ASTM) and the shaking period of 15 minutes. Particle size distribution can be determined and the mean particle size of microspheres is calculated by using the following formula

Mean particle size $=\sum$ (mean particle size of the fraction $\times$ weight fraction) $/ \sum$ (weight fraction)

\section{Density determination}

The density of the microspheres can be measured by using a multi-volume pychnometer. Accurately weighed sample in a cup, which will be placed into the multi-volume pycnometer. Helium is introduced at a constant pressure in the chamber and allowed to expand. This expansion results in a decrease in pressure within the chamber. Two consecutive reading of reduction in pressure at different initial pressure is noted. From two pressure reading the volume and density of the microspheres carrier is determined [16].

\section{Floating behavior}

The floating test on the microspheres should be carried out using the dissolution method type II apparatus, specified in the USP XXII, each jar is filled with $0.1 \mathrm{~N} \mathrm{HCl}$ solution $(\mathrm{pH} \mathrm{1.2,900} \mathrm{ml)} \mathrm{and} \mathrm{maintaining} \mathrm{the} \mathrm{temperature} \mathrm{of}$ $37 \pm 0.5^{\circ} \mathrm{C}$, which is agitated by a paddle rotated at $100 \mathrm{rpm}$. Disintegration test solution No.1 (pH 1.2), containing Tween $20(0.02 \% \mathrm{w} / \mathrm{v})$, was used as a dispersing the medium to simulate gastric fluid. After agitation for a previously determined interval, the microspheres that floated over the surface of medium and those that settled to the bottom of the flask were recovered separately. After drying, each fraction of the microspheres was weighed. The buoyancy of the microspheres was represented by the following equation [16].

\section{Angle of contact}

The angle of contact measurement determines the wetting property of a microsphere. It determines the nature of the microsphere in terms of hydrophilicity or hydrophobicity. The angle of contact measures at solid/air/water interface. The angle of contact will be measured by placing a droplet in a circular cell mounted above objective of the inverted microscope. Contact angle should be measured at $200^{\circ}$ within a minute of deposition of microsphere [16].

\section{Swelling index}

The swelling of the polymer is measured by their ability to absorb water and swells. A know desired quantity and the initial weight of the mucoadhesive microspheres are taken, followed by placing the microspheres in $0.1 \mathrm{~N} \mathrm{HCl}$, $900 \mathrm{ml}$ rotated at $75 \mathrm{rpm}$. The medium should be maintained at $37 \pm 0.5^{\circ} \mathrm{C}$ through the study. The microsphere is removed periodically from the dissolution medium. After draining free water these are measured for weight gain swelling characteristics were expressed in terms of \% water uptake

WU\% = weight of swollen microspheres - initial weight of microspheres/ initial weight of microsphere $\times 100$

\section{Percentage Yield}

The yield was calculated by the following equation

$\%$ yield $=$ weight of the mucoadhesive microspheres/weight of drug taken + total polymer weight $\times 100$

\section{Percentage drug content}

A fixed amount of microspheres contain a drug is dissolved in a suitable solvent such as ethanol, methanol, etc. by ultra-sonication the solvent is then filtered through a $0.22 \mu \mathrm{m}$ membrane filter. Finally, drug concentration was determined by the UV-visible spectrophotometrically [16].

Drug content can be calculated by the following equation formula 
$\%$ of drug content $=$ weight of drug in microspheres/weight of microspheres recovered $\times 100$

\section{The percentage of drug entrapment}

The percentage of drug entrapment can be calculated by the following equation [16].

$\%$ of drug entrapment $=$ calculated drug concentration/theoretical drug content $\times 100$

\section{Drug entrapment efficiency}

The microsphere loaded with a drug can be estimated by adding the desired volume of $0.1 \mathrm{M} \mathrm{HCl}$ solution under stirring. The mixture should have filtered with the Whatman filter paper and the amount of drug can be determined by spectrophotometrically [16].

$$
\text { Encapsulation efficiency }=\mathrm{M}(\text { actual }) / \mathrm{M} \text { (theoretical) } \times 100
$$

\section{Ex Vitro Mucoadhesive study}

The method with necessary changes. Overnight fasted male rats (200-250 g) should be sacrificed and stomach mucosa is wash with mimicking physiological saline After 15 min the mucosa held in inclined position. Mucosa was fixed to the glass slide with the cyanoacrylate glue and about desired mucoadhesive microspheres $\left(\mathrm{N}_{0}\right)$ were hydrated with the little amount of water and dispersed on the mucosal tissue and left on it for 20 min for the interaction with the mucosal surface. During this period the whole system will be placed in a constant humidity chamber which was adjusted to $90 \%$ relative humidity. At the end, the system will be washed with $0.1 \mathrm{NHCl}$ solution ( $\mathrm{pH}$ 1.2) using a peristaltic pump. Microspheres detached from the mucosa $\left(\mathrm{N}_{\mathrm{f}}\right)$ were observed visually and percent mucoadhesion was calculated [17].

$$
\% \text { mucoadhesive strength }=\mathrm{N}_{0}-\mathrm{N}_{\mathrm{f}} / \mathrm{N}_{0}
$$

\section{In Vitro drug release studies}

The drug release profiles of drug-loaded microspheres should be carried out in $0.1 \mathrm{~N} \mathrm{HCl}$ solutions $(\mathrm{pH} 1.2,900$ $\mathrm{ml}$ ) at $37 \pm 0.5^{\circ} \mathrm{C}$ using a USP XXII dissolution test apparatus. The rotation speed of the paddle was adjusted to 100 rpm for homogeneous dispersion of mucoadhesive microspheres in the dissolution medium. An aliquot should be withdrawn periodically from test solution and replaced with the same sample of fresh medium maintained at $37 \pm 0.5^{\circ} \mathrm{C}$ and assayed spectrophotometrically were collected in triplicate. In order to investigate the drug release mechanism, the release data were fitted to models representation zero-order, first-order, Higuchi and Peppas-Korsemeyer equation [17].

\section{In Vivo X-ray imaging study:}

In order to check the mucoadhesion of the microsphere, initially microspheres are fabricated by adding $50 \%$ of $\mathrm{x}$-ray grade barium sulfate to the formulation, these can be administrated through the gastric tube with the aid of 3-4 ml of water, using a syringe to push the beads forward. The position of mucoadhesive microspheres was monitored by X-ray photography [17].

\section{In Vitro wash-off test for microspheres}

The Mucoadhesive character of the microspheres should be evaluated by in-vitro adhesion testing method known as wash off method. A piece of rat stomach mucosa $1 \times 1 \mathrm{~cm}$ will be tied on to a glass slide using a thread. Approximately 100 microspheres were taken to spread on to the wet rinsed tissue specimen and the prepared slide was hung on to one of the grooves of a USP tablet disintegrating test apparatus. The disintegrating test apparatus was operated whereby the tissue specimen was given up and down movements regularly in the beaker of the disintegrating apparatus, which contained the gastric fluid ( $\mathrm{pH} \mathrm{1.2).} \mathrm{At} \mathrm{the} \mathrm{end} \mathrm{of} 30 \mathrm{~min}, 1 \mathrm{hr}$ and at hourly intervals up to 4 hrs, the number of microspheres still adhering to the tissue should be counted [17].

Percent mucoadhesion $=($ weight of adhered microspheres/weight of applied microspheres $) \times 100$

\section{Mucoadhesive Microspheres stability in the pH 1.0 HCI medium}

Mucoadhesive microspheres should be dissolved in hydrochloric acid at $37^{\circ} \mathrm{C}$, which directed that microspheres might be degraded by gastric acid. microspheres are suspended in $\mathrm{pH} 1.0 \mathrm{HCl}$ for different periods of time, when mucoadhesive microspheres are examined for any morphological changes at 1, 2, 4 and 6 hrs, by SEM studies, when there is no change in morphological character, indicated that microspheres have stability in HCl medium [17]. 


\section{Stability study}

To assess the mucoadhesive formulation stability, stability studies should be carried out as per ICH guidelines. The best mucoadhesive microspheres formulation should be selected for stability study based on in vitro drug release studies, drug entrapment efficacy and in vitro wash off test. The selected formulations should be stored at $40^{\circ} \mathrm{C} \pm 10^{\circ} \mathrm{C} /$ Ambient, $25 \pm 20^{\circ} \mathrm{C} / 60 \pm 5 \% \mathrm{RH}, 40 \pm 20^{\circ} \mathrm{C} / 75 \pm 5 \% \mathrm{RH}$ in closed high density polyethylene bottles for 90 days. The samples should be evaluated periodically for entrapment efficiency and percentage mucoadhesion, In-vitro drug release studies should be carried out $[17,18]$.

\section{Future Challenges}

The main aim of the author is to develop a factorial design by employing Design Expert (Stat ease) to the formulation, which gives best-optimized outcome data [19].

\section{Conclusion}

Here the effect of mucoadhesive microspheres may increase the gastric retention of the drug, hence increases its bioavailability. The present review attributed to mucoadhesive microspheres type of gastro retentive drug delivery systems with prominence on the novel delivery systems employing polymers. The mucoadhesive delivery system has proven to be suitable for the reduction of the transit time of the dosage form in many research works. Even though the numerous advantages offered by these delivery systems, a new technique is further required various drug to explore the in vivo data, which can mimic the in vivo performance. A mucoadhesive delivery device has wide scope in future administration of various drugs with improved adhesion and successive sustained delivery of drug throughout the gastrointestinal tract [20].

\section{Acknowledgement}

The author is thankful to guides Dr A. Kottaimuthu \& J. Venkteshwar Rao (JVR) for this valuable guidance in developing the review article, the author is also thankful to Talla Padmavathi College of Pharmacy for providing time to review this article.

\section{Conflict Of Interest}

There is no conflict of interest.

\section{References}

[1] Adeola O. Adebisi, Peter R. Laity, and Barbara R. (2014). Conway. Formulation and evaluation of floating mucoadhesive alginate beads for targeting helicobacter pylori. J. Pharm., Pharmacology, 2014, (67): 511-524.

[2] Brahma N. Singh, Kwon H. Kim. (2000). Floating drug delivery systems: an approach to oral controlled drug delivery via gastric retention. J. Control. Release, 2000, (63): 235-259.

[3] Carla M. Lopes, Catarina Bettencourt, Alessandra Rossi, Francesca Buttini, and Pedro Barata. (2016). Overview on gastroretentive drug delivery system for improving drug bioavailability. Int. J. Pharmaceutics, 2016, (510): 144-158.

[4] Dave, B. S, Amin, A. F, and Patel, M. M. (2004). Gastroretentive drug delivery system of ranitidine hydrochloride: formulation and in vitro evaluation. AAPS. Pharm. Sci. Tech., 2004, (5): 1-6.

[5] Gupta, P. K., Sau-Hung, S., and Robinson, J. (1990). Bioadhesive/mucoadhesive in drug delivery to the gastrointestinal tract bioadhesive drug delivery system. Boca Raton: CRC Press.1990, pp. 65-92.

[6] Hilton, A. K. and Deasy, P. B. (1992). In vitro and in vivo evaluation of an oral sustained-release floating dosage form of amoxicillin trihydrate. Int. J. Pharm., 1992, (86): 79-88.

[7] Hemlatha Kaurav, S. L. Hari Kumar, and Amanpreet Kaur. (2012). Mucoadhesive microspheres as carriers in drug delivery: a review. Int. J. of Drug Development \& Res., 2012, 4(2): 21-33.

[8] Hemlatha Patil, Roshan V. Tiwari, and Michael A. Repka. (2016). Recent advancements in mucoadhesive floating drug delivery system: a mini-review. J. Dr. Del. Tech., 2016, (31): 65-71.

[9] Jagtap Yogesh Mukund, Bhujbal Rohan Kantilal, and Ranpise Nisharani Sudhakar. Floating microspheres: a review. B. J. Pharm. Sci., 2012, (48): 17-30.

[10] Mobeen Mohd, Sathish, A., Prakesh, G., Banu Thaiseen, and Ramakrishna, R. (2015). Formulation and evaluation of mucoadhesive microspheres of pioglitazone hydrochloride using a natural polymer. Int. J. Pharm Sci and Res., 2015, 6(4): $722-737$.

[11] Patil, M. P., Patil, H. S., Bharat, W. T., Vinod, M. T., and Patil, V. R. (2009). Formulation and in-vitro evaluation of floating 
microspheres of acyclovir. Arch. Pharm. Sci. Res., 2009, (1): 194-198.

[12] Purnima Tripathi, U. Ubaidulla, Roopa Kishan Khar, and Vishwavibhuti. (2012). Floating drug delivery system. Int. J. Res. Dev. Pharm. L. Sci., 2012, 1(1): 1-10.

[13] Ponchel, G. and Irache, J. M. (1998). Specific and non-specific bioadhesive particulate system for oral delivery to the gastrointestinal tract. Adv. Drug Del. Rev., 1998, (34): 191-219.

[14] Raghvendra Misra and Peeyush Bhardwaj. (2016). Development and characterization of novel floating-mucoadhesive tablets bearing venlafaxine hydrochloride. Hindawai Publicorpscienti, 2016, 1-13.

[15] Shweta, A., Javed, A., Alka, A., Roop, K. K., and Sanjula, B. (2005). Floating drug delivery systems: a review. AAPS Pharm. Sci. Tech., 2005; (6): 372-390.

[16] Sarah Duggan, Wayne Cummins, Orla O. Donovan, Helenhughes, and Eleanor Owens. (2017). Thiolated polymer as mucoadhesive drug delivery systems. Euro. J. of Pharm. Sci., 2017, (100): 64-78.

[17] Surendra Ganeshlal Gattani, Pankaj Jayantilal Savaliya, Veena Shailendra Belgamwar. (2010). Floating-mucoadhesive beads of clarithromycin for the treatment of helicobacter pylori infection. Chem. Pharm. Bull., 2010; 58(6): 782-787.

[18] Shah, S. H., Patel, J. K., and Patel, N. V. (2009). Stomach specific floating drug delivery system: a review, Inter. Jour, of Pharm, tech, Research. Int. J. Pharm. Tech. Res., 2009; 1(3): 623-633.

[19] Tanwar, Y. S., Naruka, P. S., and Ojha, G. R. (2007). Development and evaluation of floating microspheres of verapamil hydrochloride. Rev. Bras. Cien. Farm., 2007, (43): 529-534.

[20] Wilding, I. R, Coupe, A. J., and Davis, S. S. (2001). The role of gamma-scintigraphy in oral drug delivery. Adv. Drug Del. Rev., 2001, (46): 103-124. 